\title{
Renal end points in clinical trials of kidney disease
}

Misghina Weldegiorgis, Dick de Zeeuw, Hiddo Lambers Heerspink

Department of Clinical Pharmacy and Pharmacology, University of Groningen, University Medical Center Groningen, Groningen, the Netherlands

Corresponding author:

H.J. Lambers Heerspink

Department of Clinical Pharmacy and Pharmacology

University Medical Center Groningen

Groningen, the Netherlands

Tel: +31 503617589

Fax: +31 503617889

E-mail: h.j.lambers.heerspink@umcg.nl

Keywords: Randomized controlled trial, nephrology, kidney disease, Glomerular Filtration Rate, Serum creatinine

Word count: 2704

Word count Abstract: 279 


\section{Purpose of review}

End-stage renal disease and doubling of serum creatinine are established hard end points in clinical trials of chronic kidney disease (CKD). These end points are debated as their accuracy and precision may not be optimal and they are late events in the progression of CKD thereby requiring large and complex trials. The purpose of the review is to examine the validity of the currently used established renal end point by comparing the end-stage renal disease part involving renal replacement therapies (dialysis or renal transplantation) and the glomerular filtration based end points involving doubling of serum creatinine.

\section{Recent findings}

Emerging data demonstrate that the RRT decision depends not only on serum creatinine but on a range of subjective factors involving a patient's wellbeing, availability of renal replacement therapies, or local guidelines. Thus, initiation of RRT is not representative of (e)GFR decline alone. On the other hand, doubling of serum creatinine reflects a sustained loss in patients starting GFR. The disadvantage of a end point based on a filtration marker is that many drugs exert opposite effects on GFR. Initially they cause a reduction in GFR followed by a stabilization of GFR decline. This ambiguous pattern complicates the interpretation of the drug effect, in particular when the end point is based on lesser declines in GFR such as $30 \%$ or $40 \%$ decline.

\section{Summary}

The currently used end points in CKD trials reflect different functions of the kidney. For the future we have to establish whether we want to characterize the effect of a novel drug on a renal filtration marker alone, on a combination of parameters involving a patient's wellbeing, or on the composite of these. 


\section{Keypoints:}

- End-stage renal disease (ESRD) and doubling of serum creatinine are established hard end points in clinical trials of chronic kidney disease (CKD)

- The ESRD endpoint involves renal replacement therapies and does not only depend on the GFR level but also on a patients' wellbeing, availability of renal replacement therapies, or local guidelines.

- The doubling serum creatinine endpoint is driven by filtration power of the kidney.

- For future trials we have to establish whether we want to characterize the drug effect on a renal filtration marker alone, on a combination of parameters involving a patient's wellbeing, or on the composite of these. 


\section{Introduction}

Chronic kidney disease (CKD) is a worldwide health problem associated with significant morbidity and mortality. In many patients progression of CKD occurs at a relentless rate. ${ }^{1}$ Treatment of CKD aims to attenuate the progressive decline in renal function in order to ultimately delay the initiation of renal replacement therapies (RRT; dialysis and renal transplantation). However, despite the increase in prevalence of CKD and the need for novel treatment strategies to mitigate the burden of the disease, few proven therapies are effective, and the residual risk remains very high. ${ }^{2}$ We clearly need to find new targets and drugs or improve the use of older ones. However, for each new drug we will have to establish the renal or cardiovascular efficacy in generally long-lasting and expensive hard outcome trials. These end points should thus be accurate (to measure what it is purporting to measure), precise (to measure with minimal variability), and reliable (duplicate independent measurements should produce the same result).

The currently established hard end point(s) in clinical trials of CKD progression is the composite of RRT and doubling of serum creatinine. Although this end point has been, and is still, used in many landmark clinical trials in renal and cardiovascular disease, ${ }^{3,4}$ it is subject to debate for several reasons. First, the individual components may not have optimal accuracy, precision and reliability. Second, the end point occurs only when renal function has substantially deteriorated requiring clinical trials of long duration or large sample sizes. Thus, in order to accrue sufficient end points within a reasonable amount of time, patients at late stages of disease with severely reduced eGFR levels and high proteinuria need to be included, whereas patients at earlier stages of disease, who may particularly benefit from the therapy, are mostly excluded. ${ }^{2,5}$ Consequently, there has been a lot of interest in the last few years to develop alternative end points which will take less long to occur with the hope to design more efficient trials in order to facilitate and accelerate the development of novel interventions for CKD.

In this review we evaluate the validity of the current composite hard renal end point and the two individual components: first, the end-stage renal disease part involving the definition of renal replacement therapies (dialysis or renal transplantation), and second, the glomerular filtration based end points including a doubling of serum creatinine or reaching a certain predefined serum creatinine value. 


\section{$\underline{\text { Renal replacement therapy as hard end point }}$}

RRT is used as a clinical end point in large intervention trials because it represents a more or less exact moment in time when the kidney fails to function. In addition, it is associated with a high burden of disease including a decreased quality of life and significant mortality. Although it is beyond doubt that the initiation of RRT has a direct impact on an individual's wellbeing and therefore represents a clinically meaningful end point, there are several drawbacks of using RRT as end point in trials. The decision to initiate RRT is not only based on an objectively defined level of serum creatinine and/or serum urea, but is also based on a subjective decision of the physician and patient. In addition, it depends on the availability of renal replacement therapies, local guidelines, health insurance of the patient, differences among doctors in interpretation of the guideline, and patient suitability for RRT. ${ }^{6,7}$ Thus in fact, RRT is a composite by itself and represents time to loss of filtration power as well as the health of the patient, and the presence of economical drivers. Thus it is no surprise that a marked variability is observed as to when to start dialysis, as recently confirmed by a questionnaire study among nephrologists in Europe. ${ }^{8}$ This variation attenuates the accuracy and precision of the RRT end point. Thus, initiation of RRT is not representative of GFR decline alone but depends on a range of subjective factors. This implies that the evaluation of a drug effect can reflect the effect of a drug on the true GFR decline but also or only on subjective factors without affecting GFR decline.

\section{Glomerular filtration based renal end points:}

Glomerular filtration has, right or wrong, been used as the ultimate measure of the function of the kidneys. GFR based parameters are therefore prominent components of a renal end point. Several have been used.

\section{Fixed serum creatinine threshold as a renal end point}

A defined serum creatinine threshold of $6 \mathrm{mg} / \mathrm{dL}(530 \mu \mathrm{mol} / \mathrm{L})$ or estimated GFR (eGFR) threshold of $15 \mathrm{ml} / \mathrm{min} / 1.73 \mathrm{~m}^{2}$ is used as the filtration component of a hard renal end point in several trials. ${ }^{9,10}$ Since the same serum creatinine value represents different eGFR values for different patients recent trials included a predefined eGFR threshold rather than a predefined serum creatinine threshold in the definition of the hard renal end point. A threshold of 15 $\mathrm{ml} / \mathrm{min} / 1.73 \mathrm{~m}^{2}$ has been chosen since it represents the threshold at which most practice guidelines recommend to initiate-start planning_RRT. ${ }^{11,12}$ The advantage of using a fixed 
serum creatinine or eGFR threshold compared to RRT is that a similar end point definition is applied to all patients as opposed to RRT initiation.

\section{Doubling of serum creatinine as a renal end point}

Doubling of serum creatinine has been accepted as a valid component of a hard renal end point. Most patients show a linear loss of renal filtration over time and thus doubling of serum creatinine reflects a sustained loss in patients starting eGFR. The doubling of serum creatinine end point is thus directly related to eGFR decline. Although the same serum creatinine value represents different eGFR values for different patients, the change in serum creatinine over time within a patient will reflect a deterioration of eGFR for that patient.

\section{$\underline{\text { Towards a lesser change in eGFR as an end point }}$}

Although the inclusion of doubling of serum creatinine or a fixed serum creatinine threshold as a component of a hard renal end point increases the event rate and power of clinical trials it is still a late event in progression of kidney disease. Therefore, it is most appropriate in clinical trials for patients with later stages of kidney disease or those with rapid progression. To examine alternative earlier renal end points for randomized controlled trials the National Kidney Foundation (NKF) in collaboration with the Food and Drug Administration (FDA) studied alternative options as described in a series of studies and reports. ${ }^{13-17}$ The first metaanalyses evaluated in a heterogeneous database of 1.7 million participants from 35 cohorts the risk of end-stage renal disease (ESRD) as a function of the percentage change in eGFR over a 2-year baseline period. ${ }^{13}$ The study demonstrated a strong and consistent association between changes in eGFR and ESRD: A 30\% decline in eGFR during a 2-year baseline period was associated with an approximately five-fold increased risk of ESRD compared to no change in eGFR whereas a 57\% decline in eGFR (equivalent to a doubling of serum creatinine) was associated with a 30-fold increased risk of ESRD. These results were confirmed and extended in a second meta-analysis of 37 randomized controlled clinical trials involving 9488 participants. ${ }^{16}$ This meta-analysis tested the associations across different treatments frequently used in the CKD population and documented a strong association between small percentage changes in eGFR and development of ESRD regardless of the intervention used, baseline proteinuria, or eGFR.

Although of interest, these observational studies are not sufficient to claim valid surrogacy. It is imperative to also assess whether the treatment effects on the surrogate (e.g. $30 \%$ or $40 \%$ eGFR decline) correlates with the treatment effect on the established end point. 
One challenge when using lesser declines in eGFR as end point is that many interventions to slow the progression of CKD, including low protein diet, renin-angiotensin-aldosteronesystem inhibitors, and endothelin receptor antagonists, differentially affect eGFR over time: initially the cause an acute fall in eGFR whereas during long-term follow-up the slow rate of eGFR decline. This ambiguous pattern complicates the interpretation of the clinical trial result when eGFR based end points are used, particularly when end points are based on small changes in GFR. Indeed, a recent post-hoc analysis of the RENAAL and IDNT trials showed that adopting lesser declines in eGFR than a 57\% (which is equivalent to a doubling of serum creatinine) increased the number of end points but due to the attenuation of the treatment effect no gain in statistical power could be documented. ${ }^{18}$ When the acute effect of the angiotensin receptor blockers tested in RENAAL and IDNT were excluded by using the month-3 eGFR value as baseline the attenuation of the treatment effect was less. This suggests that in settings where drugs have acute effects on eGFR, end points based on lesser declines in eGFR will not guarantee an improvement in statistical power. These results were confirmed in a meta-analysis of 43 studies involving 12,821 individuals. The study showed a trend towards an attenuation of the treatment effect, in particular with drugs that exert acute effects on GFR, which in turn prevented a gain in statistical power. ${ }^{15}$ It was eventually concluded that in the absence of acute eGFR effects a $40 \%$ and possibly $30 \%$ eGFR decline may be a valid end point.

\section{$\underline{\text { eGFR slope as a renal end point }}$}

An intuitively more representative end point to evaluate the effect of an intervention on renal function is to compare the slope of (e)GFR decline over time. This takes into account all the available data of a patient over time. This end point has been used in past clinical trials although it is not accepted by the regulatory agencies as hard end point to obtain drug marketing authorization. ${ }^{19-21}$ GFR slope as end point provides good statistical power under two key assumptions. First, the rate of GFR decline is constant during follow up, and second, the treatment effect does not depend on the underlying rate of GFR decline. These assumptions are frequently violated.

As discussed above, many renoprotective interventions have acute (reversible) effects on GFR in the opposite direction as the effect during long-term treatment. The acute effect can obscure the interpretation of trial results since the drug effect on the overall GFR slope may be neutral whereas the effect may be beneficial if the initial acute effect is excluded. Such ambiguity has indeed been observed in past clinical trials. For example, the effect of a 
low protein diet on overall GFR slope was neutral while low protein diet significantly slowed the chronic rate of GFR decline. ${ }^{19}$ On the other hand, the primary comparison of ramipril versus amlodipine in the AASK trial was inconclusive because the overall rate of GFR decline did not differ between the treatment arms due to the fact that amlodipine caused an initial rise in GFR. When the initial effect of amlodipine on GFR was excluded, the slope of chronic renal function decline was in fact slower in the ramipril arm. As a result of this ambiguity it is recommended that GFR or serum creatinine is measured after treatment discontinuation to verify whether a potential initial effect on GFR is reversible after treatment discontinuation. One can also calculate GFR decline from a pre-defined post-randomization visit to avoid the problems with potential acute effects on GFR. However, one should be careful since this approach violates the comparisons of randomized comparisons because the treatment groups can differ at the post-randomization time point.

Second, statistical power of (e)GFR slope analyses is compromised if the treatment effect is greater in those with a faster progression. In this situation the larger treatment effect in those with a fast progression is diluted by the smaller treatment effects in those with a moderate or slow progression. As a result, the difference between the GFR slopes attenuates and statistical power decreases. In such instances, a time-to-event analysis (e.g. time to doubling of serum creatinine) may be more powerful since these analyses reflect the treatment effect in the patients who reach the event which usually are the patients with the fastest rate of GFR decline.

\section{Issues with eGFR based end points}

Although a serum creatinine threshold (eGFR threshold) and doubling of serum creatinine are used in many large clinical trials, it should be realized that changes in serum creatinine can not only be attributed to changes in renal filtration. As GFR falls creatinine secretion increases. GFR may therefore fall without a significant increase in serum creatinine. The increase in creatinine secretion may therefore underestimate the actual GFR fall. In addition, serum creatinine and eGFR are subject to day-to-day laboratory variability. Originally, serum creatinine was measured by the alkaline-picrate (Jaffé) method. ${ }^{22}$ The Jaffé method is subject to interference from other chromogens, causing an over-estimation of serum creatinine by as much as 15 to 25\%. More recent enzymatic assays do not suffer from this problem. However, despite the use of the novel enzymatic assays, large variability in serum creatinine measurements is still reported. ${ }^{23}$ Additionally, some interventions affect serum creatinine levels without changing renal filtration. A low protein diet is a well known example which 
acutely decreases creatinine generation without affecting renal filtration. Serum creatinine levels may thus vary over time due to different factors not related to filtration and GFR. This variation may contribute to the discordance between changes in eGFR and measured GFR as recently observed in a large study of patients with type 2 diabetes. ${ }^{24}$ In this study it was shown that all GFR estimation formula failed to describe longitudinal changes in measured GFR. Collectively these data indicate that various sources of variability in serum creatinine should be considered to correctly interpret the long-term effects of interventions on an end point based on a filtration marker. To overcome some of the day-to-day variability in (e)GFR, the end point should always be sustained and confirmed by at least one subsequent measurement.

\section{What is renal function?}

We would like the renal end point to be representative of renal function. However renal function is a composite of different functions of the kidney: excretion of waste products, maintaining extracellular volume control, acid-base control, production and conversion of various hormones. The question is whether (1) we should focus on all these properties of the kidney, (2) focus only on filtration loss and use a 30\% or $40 \%$ eGFR decline as end point being aware of the potential acute eGFR effects, or (3) whether we should combine these two as is currently used in practice with the composite established end point of RRT and doubling of serum creatinine. We have to realize however that each of these options may lead to a different interpretation of the drug effect. For example, a drug that improves the tolerance of the patient to withstand the consequences of severely compromised renal function may delay the decision of RRT (patient does not complain), whereas eGFR continues to decline. If eGFR was chosen as end point, the drug effect had not been noticed despite the fact that RRT was delayed. On the other hand, a drug may worsen the symptoms of reduced renal function but does lower the GFR decline. In this example the choice of RRT would have led to a potential harmful trial results despite a beneficial effect on the rate of eGFR decline. Furthermore, when we decide to use a composite end point we should also be aware that trial results are not driven by a single component of the end point. The clinical relevance of this notion is illustrated by the results of the ACCOMPLISH renal analysis in which the trial result was driven by the eGFR component of the renal end point which in turn was heavily influenced by the acute effects of the tested interventions. ${ }^{25,}{ }^{26}$ This point highlights the need for a prespecified balance in the individual components of the renal end point.

\section{Conclusions}


For future trials, we have to establish whether we want to characterize the effect of a novel drug on a renal filtration marker, on a combination of parameters involving a patient's wellbeing or their combination. To make such a decision, a worldwide consensus meeting is required involving all stakeholders (industry, regulators, clinicians, patients, health-payers).

We propose that we use the currently established hard end point of doubling of serum creatinine, RRT, renal death. In order to facilitate current clinical trials and development of new interventions we suggest allowing conditional approvals (market authorizations) on softer end points like albuminuria, 30\% eGFR change, or eGFR slope difference. This should be subsequently confirmed in hard end point trials in which the composite endpoint is properly balanced to represent the multiple aspects of renal function.

\section{Acknowledgements:}

None

Financial Support and Sponsorship:

None

\section{Conflicts of interest}

Dick de Zeeuw is consultant for and received honoraria (to employer) from AbbVie, Astellas, AstraZeneca, BMS, Chemocentryx, J\&J, Hemocue, Novartis, Reata, Takeda, Vitae. Hiddo Lambers Heerspink is consultant for and received honoraria (to employer) from AbbVie, Astellas, J\&J, Reata. Misghina Weldegiorgis has no conflicts of interests. 


\section{References:}

- References of interest

$\bullet \bullet$ References of special interest

1. Levey AS, Coresh J. Chronic kidney disease. Lancet. Jan 14 2012;379(9811):165-180.

2. Heerspink HJ, de Zeeuw D. The kidney in type 2 diabetes therapy. Rev Diabet Stud. Fall 2011;8(3):392-402.

3. Brenner BM, Cooper ME, de Zeeuw D, Keane WF, Mitch WE, Parving HH, et al. Effects of losartan on renal and cardiovascular outcomes in patients with type 2 diabetes and nephropathy. N Engl J Med. Sep 20 2001;345(12):861-869.

4. Parving HH, Brenner BM, McMurray JJ, de Zeeuw D, Haffner SM, Solomon SD, et al. Cardiorenal end points in a trial of aliskiren for type 2 diabetes. $N$ Engl $J$ Med. Dec 6 2012;367(23):2204-2213.

5. Palmer AJ, Annemans L, Roze S, Lamotte M, Lapuerta P, Chen R, et al. Costeffectiveness of early irbesartan treatment versus control (standard antihypertensive medications excluding ACE inhibitors, other angiotensin-2 receptor antagonists, and dihydropyridine calcium channel blockers) or late irbesartan treatment in patients with type 2 diabetes, hypertension, and renal disease. Diabetes Care. Aug 2004;27(8):1897-1903.

6. Caskey FJ, Kramer A, Elliott RF, Stel VS, Covic A, Cusumano A, et al. Global variation in renal replacement therapy for end-stage renal disease. Nephrol Dial Transplant. Aug 2011;26(8):2604-2610.

7. Couchoud C, Guihenneuc C, Bayer F, Lemaitre V, Brunet P, Stengel B. Medical practice patterns and socio-economic factors may explain geographical variation of end-stage renal disease incidence. Nephrol Dial Transplant. Jun 2012;27(6):23122322.

8. $\quad$ van de Luijtgaarden MW, Noordzij M, Tomson C, Couchoud C, Cancarini G, Ansell $\mathrm{D}$, et al. Factors influencing the decision to start renal replacement therapy: results of a survey among European nephrologists. Am J Kidney Dis. Dec 2012;60(6):940-948.

This survey showed that only for uncomplicated patients half of the nephrologists considered excretory function the most important factor for the decision to start RRT.

9. Lewis EJ, Hunsicker LG, Clarke WR, Berl T, Pohl MA, Lewis JB, et al. Renoprotective effect of the angiotensin-receptor antagonist irbesartan in patients with nephropathy due to type 2 diabetes. N Engl J Med. Sep 20 2001;345(12):851-860.

10. Imai E, Chan JC, Ito S, Yamasaki T, Kobayashi F, Haneda M, et al. Effects of olmesartan on renal and cardiovascular outcomes in type 2 diabetes with overt nephropathy: a multicentre, randomised, placebo-controlled study. Diabetologia. Dec 2011;54(12):2978-2986.

11. Tattersall JE, Pedrini L, Martin-Malo AM. When to start dialysis? Nephrol Dial Transplant. 2002;17(Suppl 10):10.

12. Levin A, Hemmelgarn B, Culleton B, Tobe S, McFarlane P, Ruzicka M, et al. Guidelines for the management of chronic kidney disease. Cmaj. Nov 18 2008;179(11):1154-1162.

13. - Coresh J, Turin TC, Matsushita K, Sang Y, Ballew SH, Appel LJ, et al. Decline in estimated glomerular filtration rate and subsequent risk of end-stage renal disease and mortality. Jama. Jun 25 2014;311(24):2518-2531. 
This article describes the strong and consistent association between a 30\% and $40 \%$ decline in eGFR and subsequent renal and mortality outcomes.

14. $\bullet$ Levey AS, Inker LA, Matsushita K, Greene T, Lawrence J, Willis K, et al. GFR Decline as an Endpoint for Clinical Trials in CKD: A Scientific Workshop Sponsored by the National Kidney Foundation and the US Food and Drug and Administration. Am J Kidney Dis. 2014:64(6):821-35.

This workshop report reviews the data, discussions, and conclusions of the NKF-FDA workshop on GFR decline as end point in clinical trials of chronic kidney disease.

15. $\bullet$ Inker LA, Lambers Heerspink HJ, Mondal H, Smith CH, Noubary F, Coresh J, et al. GFR decline as an alternative endpoint for kidney failure: a meta analysis of treatment effects from 37 randomized trials. 2014 ;64(6):849-59..

This meta-analyses compared the consistency of the treatment effects of various interventions used in CKD patients on established renal endpoints with alternative renal endpoints defined by a eGFR decline of $30 \%$ or $40 \%$

16. Lambers Heerspink HJ, tighiouart H, Sang Y, Ballew S, Mondal H, Matsushita K, et al. GFR decline and subsequent risk of established kidney outcomes - a meta-analysis of 37 randomized controlled trials. Am J Kidney Dis. 2014;64(6):860-6.

17. Greene T. GFR decline as an alternative endpoint for kidney failure - A Simulation Study of the Validity and Utility of eGFR-Based Surrogate Time-to-Event Endpoints in Chronic Kidney Disease. Am J Kidney Dis. 2014:64(6):867-79.

This simulation study assessed sample sizes and type 1 error rates for clinical trials using a $30 \%$ or $40 \%$ eGFR decline as end point. The study concluded that in the presence of acute effects the risk for type 1 error is increased and therefore careful consideration of the drug characteristics is required before using these endpoints.

18. - Lambers Heerspink HJ, Weldegiorgis $M$, Inker LA, Gansevoort R, Parving HH, Dwyer JP, et al. Estimated GFR Decline as a Surrogate End Point for Kidney Failure: A Post Hoc Analysis From the Reduction of End Points in Non-Insulin-Dependent Diabetes With the Angiotensin II Antagonist Losartan (RENAAL) Study and Irbesartan Diabetic Nephropathy Trial (IDNT). Am J Kidney Dis. 2014:63(2):244-50. This was the first article that evaluated the performance of clinical trial end points defined by lesser declines in eGFR than a halving of baseline eGFR (doubling of serum creatinine). The study showed that eGFR declines lesser than a doubling of serum creatinine did not consistently increase statistical power due to the attenuation of treatment effects.

19. Klahr S, Levey AS, Beck GJ, Caggiula AW, Hunsicker L, Kusek JW, et al. The effects of dietary protein restriction and blood-pressure control on the progression of chronic renal disease. Modification of Diet in Renal Disease Study Group. $N$ Engl $J$ Med. Mar 31 1994;330(13):877-884.

20. Wright JT, Jr., Bakris G, Greene T, Agodoa LY, Appel LJ, Charleston J, et al. Effect of blood pressure lowering and antihypertensive drug class on progression of hypertensive kidney disease: results from the AASK trial. Jama. Nov 20 2002;288(19):2421-2431.

21. Barnett AH, Bain SC, Bouter P, Karlberg B, Madsbad S, Jervell J, et al. Angiotensinreceptor blockade versus converting-enzyme inhibition in type 2 diabetes and nephropathy. N Engl J Med. Nov 4 2004;351(19):1952-1961.

22. Bonsnes RW TH. On the colorimetric determination of creatinine by Jaffe reaction. $J$ Biol Chem. 1945;158:581-591. 
23. Joffe M, Hsu CY, Feldman HI, Weir M, Landis JR, Hamm LL. Variability of creatinine measurements in clinical laboratories: results from the CRIC study. Am $J$ Nephrol.31(5):426-434.

24. Gaspari F, Ruggenenti P, Porrini E, Motterlini N, Cannata A, Carrara F, et al. The GFR and GFR decline cannot be accurately estimated in type 2 diabetics. Kidney Int. Jul 2013;84(1):164-173.

25. Bakris GL, Sarafidis PA, Weir MR, Dahlof B, Pitt B, Jamerson K, et al. Renal outcomes with different fixed-dose combination therapies in high-risk hypertensive patients. Lancet. 2010.

26. Lambers Heerspink HJ, de Zeeuw D. Composite renal endpoints: was ACCOMPLISH accomplished? Lancet. Apr 3 2010;375(9721):1140-1142. 